\section{FRI0036 ASSOCIATION BETWEEN THE 18FDG-PET IMAGING AND THE PATHOLOGICAL FINDINGS OF RHEUMATOID SYNOVITIS}

C. Okura, Y. Yonemoto, K. Okamura, T. Suto, M. Tachibana, H. Chikuda. Orthopaedic Surgery, Gunma University Graduate School of Medicine, Maebashi, Japan

Background: It has been reported that 18FDG-PET (PET) is useful in the evaluation of RA and for monitoring the effects of treatment on the disease activity, so its utility for the evaluation of arthritis is expected. However, the mechanism underlying the uptake of FDG into the inflamed joint is still unclear. Objectives: The aim of this study was to investigate the associations among the amount of FDG uptake in RA joints, the inflammatory findings with regard to the pathology of the synovium and the clinical findings.

Methods: We performed PET in 18 RA patients who underwent Total Knee Arthroplasty surgery in our hospital just prior to surgery. We calculated the FDG uptake as the standardized uptake value (SUV)max and scored it using the Rooney score, with the degree of inflammation of the synovial tissues used for the pathological evaluation. We evaluated the associations among the SUVmax, Rooney score, CRP level, ESR and MMP-3 level just before surgery.

Results: The subjects were 18 cases with 20 joints, which were in four females and 14 males. At the time of surgery, the average age of the patients was $66.7 \pm 7.9$ years old, and the mean disease duration was $20.8 \pm 14.0$ years. Significant correlation was not observed between SUVmax and total Rooney score $(r=0.056, p=0.814)$. But there were strong correlations between SUVmax and some of the individual items in Rooney score, including the "Synoviocyte hyperplasia", and also between SUV max and "Diffuse infiltrates of lymphocytes" $(r=0.512, p=0.021$ and $r=0.581, p=0.007$, respectively).

Conclusions: The accumulation of FDG was associated with the extent of "synoviocyte hyperplasia" and "diffuse infiltrates of lymphocytes". It is estimated that accumulation of FDG is associated with relatively early stage of active inflammation of RA.

References:

[1] McQueen FM, et al. Clin Rheumatol: 2007.

[2] Beckers C, et al. Eur J Nucl Med Mol Imaging; 2006.

[3] Okamura K, et al. Rheumatology (Oxford); 2012.

Disclosure of Interest: None declared

DOI: 10.1136/annrheumdis-2017-eular.7021

\section{FRI0037 MER-MEDIATED EFFEROCYTOSIS TEMPERS ARTHRITIS BY PREVENTING NEUTROPHILS TO GO INTO SECONDARY NECROSIS AND SPILL THEIR INFLAMMATORY CONTENT IN THE JOINT}

C.E.J. Waterborg, S. Beermann, M.B. Bennink, M.I. Koenders, P.L.E.M. van Lent, F. van den Hoogen, P.M. van der Kraan, F.A.J. van de Loo. Experimental Rheumatology, Radboudumc, Nijmegen, Netherlands

Background: Rheumatoid arthritis is characterized by an inflammatory response in synovial joints, showing a predominant influx of neutrophils. These cells are cytotoxic and contribute to matrix degradation. In addition, they are implicated as a source of citrullinated auto-antigens, leading to the production of anti-citrullinated protein antibodies. Neutrophils have a relative short life span and many of them undergo apoptosis. If they are not cleared, they undergo secondary necrosis and release their cell content. A key mediator in the resolution of inflammation and the uptake of apoptotic cells, or efferocytosis, is the receptor tyrosine kinase Mer.

Objectives: To elucidate the local role of Mer during gonarthritis.

Methods: Macrophages were transduced by adenoviruses encoding the Mer ligand Pros1 or Luciferase. One day after the collagen booster injection in mice, Mer-specific antibodies or IgG antibodies, or adenoviruses overexpressing Pros1 or Luciferase were injected intravenously. Mice were euthanized at day 30 or 36 , respectively. The KRN serum transfer arthritis model was induced by two intraperitoneal injections of arthritic $\mathrm{K} / \mathrm{BxN}$ serum in either Mer-deficient or wild-type (WT) mice, or in mice that overexpress Pros1 or Luciferase in their knee joints. Mice were euthanized at day 7 or day 14, respectively. From all mice, serum was taken for cytokine profiling and knee joints were isolated for either synovial gene expression or histology and immunohistochemistry.

Results: Adenoviral overexpression of the Mer ligand Pros1 resulted in reduced production of pro-inflammatory cytokines and chemokines by macrophages, compared to Luciferase. In addition, local Pros1 overexpression resulted in reduced expression of pro-inflammatory and pro-destructive mediators by synovial cells of arthritic mice. Systemic and local Pros1 overexpression diminished joint pathology, reduced the number of cleaved Caspase 3-positive apoptotic cells and secondary necrotic neutrophils. Conversely, inhibiting Mer-mediated efferocytosis by either Mer-specific antibodies or Mertk gene ablation resulted in aggravation of arthritis compared to controls, as evidenced by increased inflammation and tissue destruction. Additionally, Mer-inhibited mice had increased numbers of apoptotic cells in their knee joints, and higher serum levels of IL-16C, a cytokine released by secondary necrotic neutrophils.

Conclusions: Together, these results demonstrate that Mer locally plays a unique protective role in knee joint disease by enhancing resolution of arthritis. Our data suggest that promoting and/or restoring Mer-mediated uptake of apoptotic cells in the arthritic joint might be therapeutically beneficial.

Disclosure of Interest: None declared
DOI: 10.1136/annrheumdis-2017-eular.4902

\section{FRI0038 RELEASE OF PEPTIDYLARGININE DEIMINIASE 2 FROM ACTIVATED NEUTROPHILS}

M.A. Steffensen, D. Damgaard, M. Bassi, C.H. Nielsen. Institute for Inflammation Research, Rigshospitalet, Copenhagen University Hospital, Copenhagen, Denmark

Background: Extracellular citrullination catalyzed by peptidylarginine deiminase (PAD) is thought to play a central role in the pathogenesis of rheumatoid arthritis. Neutrophils are a major reservoir of PAD2 and PAD4. Cellular release of PAD2 and PAD4 is usually considered a consequence of cell death.

Objectives: We aimed to determine if PAD2 can be released from live, activated neutrophils as an active process.

Methods: Whole blood cells were purified from healthy blood were stimulated with phorbol 12-myristate 13-acetate (PMA). To capture PAD2 released from neutrophils and detect it by flow cytometry, we used biotinylated anti-CD15 and anti-PAD2 (mAb DN6) mAbs linked by streptavidin, and a different PE-labelled anti-PAD2 amtibody (mAb DN2). In addition, intracellular PAD2 was quantified by intracellular staining with PE-anti-PAD2. PAD2 released from leukocytes and subcellular fractions of human granulocytes were assessed for content of PAD2 using an in-house luminex-based assay.

Results: On incubation of whole blood cells with PMA, PAD2 was detectable in the supernatants after 30 minutes, and levels increased thereafter in parallel with increasing cell death. However, using PAD2 catch reagent, we found that live neutrophils released PAD2 in the 30 minutes after stimulation. Intracellular staining for PAD2 showed that the content of PAD2 in live neutrophils decreased correspondingly. Upon subcellular fractioning of granulocytes, the majority of PAD2 was found in cytosol and, in 25 times lower quantities, in the fraction containing plasma membranes and secretory vesicles. Sparse amounts of PAD2 were also observed in gelatinase granules.
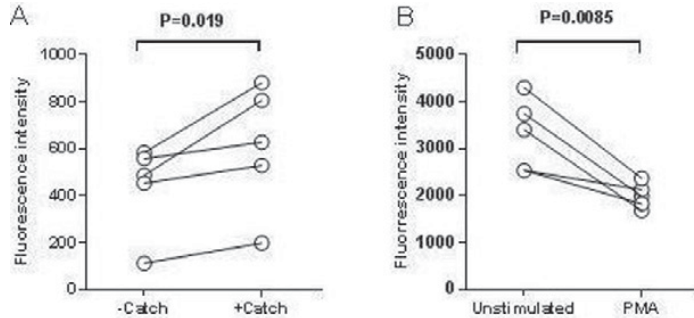

Figure 1: Release of PAD2 by live ne utrophils following activation. (A) Whole blood cells were stimulated with PMA for 30 minutes in presence of a catch reagent consisting of co-conjugated anti-CD15 and anti-PAD2 antibodies, and PAD2 caught on the surface of CD15-granulocytes was de tected by flow cytometry using PE-conjug ated anti-PAD2 antibody ( + Catch). Cells not exposed to catch reagent (-Catch) were included as controls. (B) Whole blood cells were incubated with or without PMA for 30 minutes, and the conten of PAD2 in granulocytes was assessed using flow cytometry with a PE-conjugated anti-PAD2 antibody (DN2). In both A and B, granulocytes were gated on the basis of forward and side light scatter characteristics, and dead cells staining positive for a near-IR forward and side light scatter char

Conclusions: In conclusion, PAD2 can be released from live, activated neutrophils, which may contribute to extracellular citrullination and thereby play a role in driving inflammatory processes in RA patients with immune responses to citrullinated proteins.

Disclosure of Interest: None declared

DOI: 10.1136/annrheumdis-2017-eular.5964

\section{FRI0039 ENDOTHELIAL DYSFUNCTION IN RHEUMATOID ARTHRITIS: WHICH EFFECT OF METHOTREXATE? A STUDY IN ADJUVANT INDUCED ARTHRITIS MODEL}

R. Bordy ${ }^{1}$, P. Totoson ${ }^{1}$, F. Verhoeven ${ }^{2}$, C. Prati ${ }^{1,2}$, J. Moretto $^{1}$,

M. Tournier-Nappey ${ }^{1}$, C. Demougeot ${ }^{1}$. ${ }^{1}$ Pepite Ea4267, Université de Franche-Comté; ${ }^{2}$ Rhumatologie, CHRU Besançon, Besançon, France

Background: Rheumatoid arthritis (RA) is associated with increased cardiovascular (CV) risk [1] secondary to endothelial dysfunction (ED) [2]. There is accumulating evidence that methotrexate (MTX), first intention DMARD, reduces $\mathrm{CV}$ risk in RA [3], but the mechanisms involved are still unknown.

Objectives: The aim of this study was to determine the effect of MTX on endothelial function in arthritis and to investigate its effect on endothelial pathways.

Methods: Experiments were conducted in the adjuvant-induced arthritis (AIA) model in Lewis rat. At onset of arthritis, rats were treated by a sub-cutaneous injection of MTX (1 mg/kg/week) or phosphate buffer saline (vehicle) for 3 weeks. Arthritis score was daily monitored. At the end of treatment, thoracic aorta was harvested to measure the relaxation to acetylcholine on pre-constricted aortic rings in the presence or not of inhibitor of nitric oxide (NO) synthase (L-NAME), arginase (nor-NOHA), EDHF (Apamin/Charybdotoxin), or a superoxide dismutase analog (Tempol). The effect of norepinephrine (NE) and sodium nitroprusside 